Advanced Graph Theory and Combinatorics 
To Christelle, Aurore and Maxime 
Series Editor

Valérie Berthé

\section{Advanced Graph Theory and Combinatorics}

Michel Rigo

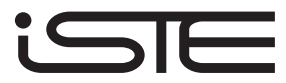


First published 2016 in Great Britain and the United States by ISTE Ltd and John Wiley \& Sons, Inc.

Apart from any fair dealing for the purposes of research or private study, or criticism or review, as permitted under the Copyright, Designs and Patents Act 1988, this publication may only be reproduced, stored or transmitted, in any form or by any means, with the prior permission in writing of the publishers, or in the case of reprographic reproduction in accordance with the terms and licenses issued by the CLA. Enquiries concerning reproduction outside these terms should be sent to the publishers at the undermentioned address:

ISTE Ltd

27-37 St George's Road

London SW19 4EU

UK

www.iste.co.uk
John Wiley \& Sons, Inc.

111 River Street

Hoboken, NJ 07030

USA

www.wiley.com

\section{(C) ISTE Ltd 2016}

The rights of Michel Rigo to be identified as the author of this work have been asserted by him in accordance with the Copyright, Designs and Patents Act 1988.

Library of Congress Control Number: 2016953238

British Library Cataloguing-in-Publication Data

A CIP record for this book is available from the British Library

ISBN 978-1-84821-616-7 


\section{Contents}

Foreword $\ldots \ldots \ldots \ldots \ldots \ldots \ldots \ldots \ldots$ ix

Introduction $\ldots \ldots \ldots \ldots \ldots \ldots \ldots \ldots \ldots \ldots \ldots \ldots \ldots \ldots$

Chapter 1. A First Encounter with Graphs . . . . . . . . . 1

1.1. A few definitions . . . . . . . . . . . . . . . . . . 1

1.1.1. Directed graphs . . . . . . . . . . . . . 1

1.1.2. Unoriented graphs . . . . . . . . . . . . . . . . . 9

1.2. Paths and connected components . . . . . . . . . . . 14

1.2.1. Connected components . . . . . . . . . . . . . . 16

1.2.2. Stronger notions of connectivity . . . . . . . . . . 18

1.3. Eulerian graphs . . . . . . . . . . . . . . . . 23

1.4. Defining Hamiltonian graphs . . . . . . . . . . . . . 25

1.5. Distance and shortest path _. . . . . . . . . . . . 27

1.6. A few applications . . . . . . . . . . . . . . . . 30

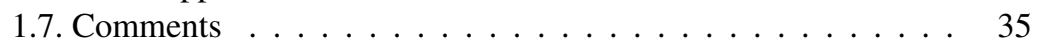

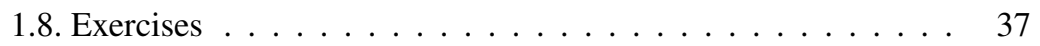

Chapter 2. A Glimpse at Complexity Theory . . . . . . . . . . . 43

2.1. Some complexity classes . . . . . . . . . . . . . . . 43

2.2. Polynomial reductions . . . . . . . . . . . . . . 46

2.3. More hard problems in graph theory . . . . . . . . . . . . 49

Chapter 3. Hamiltonian Graphs $\ldots \ldots \ldots \ldots$

3.1. A necessary condition . . . . . . . . . . . . . 53

3.2. A theorem of Dirac . . . . . . . . . . . . . . 55 
3.3. A theorem of Ore and the closure of a graph . . . . . . . . 56

3.4. Chvátal's condition on degrees . . . . . . . . . . . . . 59

3.5. Partition of $K_{n}$ into Hamiltonian circuits . . . . . . . . . . 62

3.6. De Bruijn graphs and magic tricks . . . . . . . . . . . . . 65

3.7. Exercises ..................... . . 68

Chapter 4. Topological Sort and Graph Traversals _ . . . . . 69

4.1. Trees . . . . . . . . . . . . . . . . . . . 69

4.2. Acyclic graphs . . . . . . . . . . . . . . . . . . . . . . 79

4.3. Exercises ....................... 82

Chapter 5. Building New Graphs from Old Ones . . . . . . . . . 85

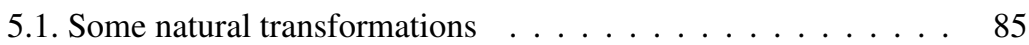

5.2. Products . . . . . . . . . . . . . . . . 90

5.3. Quotients .......................... 92

5.4. Counting spanning trees . . . . . . . . . . . . . . . . . . 93

5.5. Unraveling . . . . . . . . . . . . . . . . . . . . . . . . . . . . . . . . . . . 94

5.6. Exercises . . . . . . . . . . . . . . . 96

Chapter 6. Planar Graphs . . . . . . . . . . . . . . . . . . . . . . 99

6.1. Formal definitions . . . . . . . . . . . . . . . . . . . . . . . 99

6.2. Euler's formula . . . . . . . . . . . . . . . . . . . . . 104

6.3. Steinitz' theorem . . . . . . . . . . . . . . . . . . . 109

6.4. About the four-color theorem . . . . . . . . . . . . . . . . . . . . . . . . . 113

6.5. The five-color theorem . . . . . . . . . . . . . . 115

6.6. From Kuratowski's theorem to minors . . . . . . . . . . . . 120

6.7. Exercises . . . . . . . . . . . . . . . . 123

Chapter 7. Colorings . . . . . . . . . . . . . . . . . . . . . 127

7.1. Homomorphisms of graphs . . . . . . . . . . . . . . . 127

7.2. A digression: isomorphisms and labeled vertices . . . . . . . . 131

7.3. Link with colorings . . . . . . . . . . . . . . . . . . 134

7.4. Chromatic number and chromatic polynomial . . . . . . . . 136

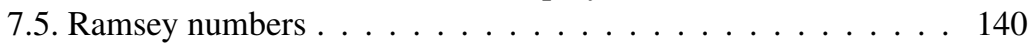

7.6. Exercises . . . . . . . . . . . . . . . . . 147

Chapter 8. Algebraic Graph Theory _. . . . . . . . . . . 151

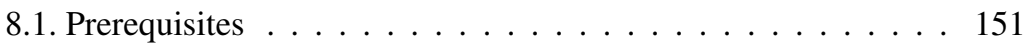

8.2. Adjacency matrix . . . . . . . . . . . . . . . . 154

8.3. Playing with linear recurrences . . . . . . . . . . . . . . . . 160 
8.4. Interpretation of the coefficients . . . . . . . . . . . . . . . . 168

8.5. A theorem of Hoffman . . . . . . . . . . . . . . . . . . 169

8.6. Counting directed spanning trees . . . . . . . . . . . . . . . . 172

8.7. Comments . . . . . . . . . . . . . . . . . . 177

8.8. Exercises ..................... 178

Chapter 9. Perron-Frobenius Theory _. . . . . . . . . . 183

9.1. Primitive graphs and Perron's theorem . . . . . . . . . . . 183

9.2. Irreducible graphs . . . . . . . . . . . . . . . . . . . . . . . . . . . . . . . . . . . . . . . . . . . . . .

9.3. Applications . . . . . . . . . . . . . . . . . . . . . . . . . . . . . . . . . . . . . . . . . . .

9.4. Asymptotic properties . . . . . . . . . . . . . . . . . 195

9.4.1. Canonical form . . . . . . . . . . . . . . . . . 196

9.4.2. Graphs with primitive components . . . . . . . . . . . . . . 197

9.4.3. Structure of connected graphs . . . . . . . . . . . . . 206

9.4.4. Period and the Perron-Frobenius theorem . . . . . . . . 214

9.4.5. Concluding examples . . . . . . . . . . . . . . . . . 218

9.5. The case of polynomial growth . . . . . . . . . . . . . . . . . . . . . . . . . . . 224

9.6. Exercises ........................ 231

Chapter 10. Google's Page Rank . . . . . . . . . . . . . 233

10.1. Defining the Google matrix . . . . . . . . . . . . . . 238

10.2. Harvesting the primitivity of the Google matrix . . . . . . . . 241

10.3. Computation . . . . . . . . . . . . . . . 246

10.4. Probabilistic interpretation . . . . . . . . . . . . . . 246

10.5. Dependence on the parameter $\alpha$. . . . . . . . . . . 247

10.6. Comments . . . . . . . . . . . . . . . . . . . 248

Bibliography . . . . . . . . . . . . . . . . . . . . . 249

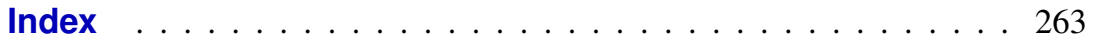




\section{Foreword}

The book content reflects the (good) taste of the author for solid mathematical concepts and results that have exciting practical applications. It is an excellent textbook that should appeal to students and instructors for its very clear presentation of both classical and more recent concepts in graph theory.

Vincent BLONDEL

Professor of Applied Mathematics, University of Louvain

September 2016 


\section{Introduction}

This book is a result of lecture notes from a graph theory course taught at the University of Liège since 2005. Through the years, this course evolved and lectures were given at different levels ranging from second-year undergraduates in mathematics to students in computer science entering master's studies. It was therefore quite challenging to find a suitable title for this book.

\section{Advanced or not so advanced material?}

I hope that the reader will not feel cheated by the title (which is always tricky to choose). In some aspects, the material is rather elementary: we will start from scratch and present basic results on graphs such as connectedness or Eulerian graphs. In the second part of the book, we will analyze in great detail the strongly connected components of a digraph and make use of Perron-Frobenius theory and formal power series to estimate asymptotics on the number of walks of a given length between two vertices. Topics with an algebraic or a combinatorial flavor such as Ramsey numbers, introduction to Robertson-Seymour theorem or PageRank can be considered as more advanced.

In the history of mathematics, we often mention the seven bridges of Königsberg problem as the very first problem in graph theory. It was studied by the famous mathematician L. Euler in 1736. It took two centuries to develop and build a complete theory from a few scattered results. Probably the first book on graphs is Theorie der endlichen und unendlichen Graphen [KÖN 90] written by the Hungarian mathematician D. König in 1936, a student of J. Kürschák and H. Minkowski. In the middle of the 20th Century, graph theory matured into a well-defined branch of discrete mathematics and 
combinatorics. We observe many mathematicians turning their attention to graph theory with books by C. Berge, N. Biggs, P. Erdős, C. Kuratowski, W. T. Tutte, K. Wagner, etc. We have seen an important growth during the past decades in combinatorics because of the particular interactions existing with optimization, randomized algorithms, dynamical programming, ergodic or number theory, theoretical computer science, computational geometry, molecular biology, etc. On MathSciNet, if you look for research papers with a Primary Mathematics Subject Classification equal to 05C (which stands for graph theory and is divided into 38 subfields ranging from planar graphs to connectivity, random walks or hypergraphs), then we find for the period 2011-2015 between 3, 300 and 3, 700 papers published every single year.

In less than a century, many scientists and entrepreneurs have seen the importance of graph theory in real-life applications. In a recent issue of Wired magazine (March 2014), we can read an article entitled Is graph theory a key to understanding big data? by R. Marsten. Let us quote his conclusion: "The data that we have today, and often the ways we look at data, are already steeped in the theory of graphs. In the future, our ability to understand data with graphs will take us beyond searching for an answer. Creating and analyzing graphs will bring us to answers automatically". Later, in the same magazine (May 2014), E. Eifrem wrote: "We're all well aware of how Facebook and Twitter have used the social graph to dominate their markets, and how Facebook and Google are now using their Graph Search and Knowledge Graph, respectively, to gear up for the next wave of hyper-accurate and hyper-personal recommendations, but graphs are becoming very widely deployed in a host of other industries".

This book reflects the tastes of the author but also includes some important applications such as Google's PageRank. It is only assumed that the reader has a working knowledge of linear algebra. Nevertheless, all the definitions and important results are given for the sake of completeness. The aim of the book is to provide the reader with the necessary theoretical background to tackle new problems or to easily understand new concepts in graph theory. Algorithms and complexity theory occupy a very small portion of the book (mostly in the first chapters).

\section{This book, others and inspiration}

Many other books on graphs do exist and the reader should not limit himself or herself to a single source. The Internet is also a formidable resource. Even if we have to be cautious when looking for information on the Web, Wikipedia contains a wealth of relevant information (but keep a critical 
eye). The present book starts with some unavoidable general material, then moves on to some particular topics with a combinatorial flavor. Powers of the adjacency matrix and Perron theory have a predominant role. The reader could probably start with this book and then move to [BRU 11] as a good companion to get a deeper knowledge of the links between linear algebra and graphs. See also [BAP 14] or the classical [GOD 01] in algebraic graph theory. Similarly, a comprehensive presentation of PageRank techniques can be found in [LAN 06]. The authors of that book, A. Langville and C. D. Meyer, also specialized in ranking techniques (see [LAN 12]). Another general reference discussing partially the same topics as here - and I do hope, with the same philosophy - is by R. Diestel where much more material and a particular emphasis on infinite graphs may be found. The present book is smaller and is thus well suited for readers who do not want to spend too much time on a specialized topic.

Having given a graph theory course for more than 10 years, I'm probably unconsciously tempted to take ownership of the proofs that I found somewhere else. It is no easy task to cite and give credits to all the sources that inspired me in this process. Let me mention [BIG 93] for algebraic aspects and chromatic polynomial, [TUT 01] for its first chapters and [ORE 90] for planar graphs and his proof of the 5-color theorem. I should also mention [BOL 98] (with an impressive collection of exercises) and [BER 89]. Finally, I remember that projects available on the Web and run by D. Arnold (College of Redwoods) were inspiring.

\section{Practical organization}

For a one-semester course, here is the time I usually devote to selected topics with 15 lectures of $90 \mathrm{~min}$. Moreover, sessions for exercises take the same amount of time. The book contains extra material and more than 115 exercises:

- digraphs, paths, connected components (sections 1.1 and 1.2);

- Eulerian graphs, distance and shortest path (sections 1.3 and 1.5);

- introduction to Hamiltonicity, applications (sections 1.4 and 1.6);

- trees (section 4.1);

- homomorphisms, group of automorphisms (sections 7.1 and 7.2);

- Hamiltonian graphs (sections 3.1-3.4);

- topological sort (Chapter 4); 
- adjacency matrix, counting walks (sections 8.2 and 8.3);

- primitivity, Perron's theorem and asymptotics (sections 9.1 and 9.4);

- irreducibility and asymptotics (sections 9.2 and 9.4);

- applications of Perron-Frobenius theory (section 9.3);

- Google's PageRank (Chapter 10);

- planar graphs and Euler's formula (sections 6.1-6.3);

- colorings, the five-color theorem (section 6.5);

- Ramsey numbers (section 7.4).

Definitions are emphasized and the most important ones are written in bold face, so that definitions of recurrent notions can be found more easily. Labels of bibliographic entries are based on the first three letters of the last name of the first author and then the year of publication. In the bibliography, entries are sorted in alphabetical order using these labels.

I address special thanks to Émilie Charlier, Aline Parreau and Manon Stipulanti for their great feedback leading to some major improvements in this book. Of course, Valérie Berthé always plays a special role. I am very pleased to blame her (indeed, this adventure produced some pressure from time to time) for what this project finally looks like. She is always supportive and enthusiastic. I also thanks several colleagues: Benoît Donnet, Eric Duchêne, Fabien Durand, Narad Rampersad, Eric Rowland and Élise Vandomme for the valuable time they spent reading drafts of parts of this book. I will not forget Laurent Waxweiler who gave and prepared the very first exercise classes for my course. I also thank my many students along the years. Their questions, queries and enthusiasm allowed me to improve, over the time, the overall presentation and sequencing of this book.

Michel RIGO

September, 2016 